\title{
Tieline Controls in Microgrid Applications
}

\author{
M. Adamiak \\ GE Digital Energy, Multilin
}

K.Bahei-Eldin
GE Global Research,

\author{
S.Bose, \\ GE Global Research,
}

\author{
Y.Liu, \\ GE Global Research,
}

\section{Introduction}

As electric distribution technology moves into the next century, many trends are becoming apparent that will change the requirements of energy delivery. These changes are being driven from both the demand side where higher energy availability and efficiency are desired, and from the supply side where the integration of distributed generation and peak-shaving technologies must be accommodated. Distribution systems possessing distributed generation and controllable loads with the ability to operate in both grid-connected and standalone modes are an important class of the so-called Microgrid power system (Figure 1).

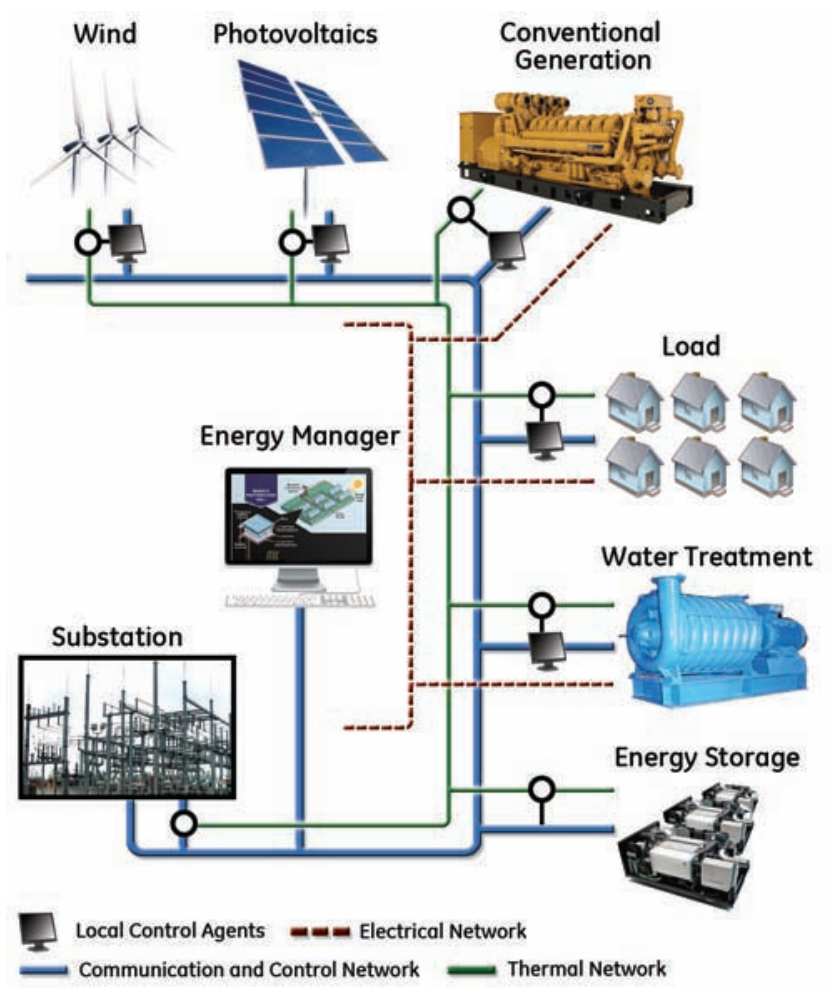

Figure 1.

Microgrid power system

This class of Microgrid strives for optimized operation of the aggregated distribution system by coordinating the distributed generation and load resources - not only when connected to the main grid but also in a stand-alone mode. In either mode of operation, advanced local controls, energy management and protection technologies are required for robustness and reliability.

While the energy management optimization objective function can be tailored to the needs of each application, in general the overall objective is to optimize operating performance and cost in the normally grid-connected mode, while ensuring that the system is capable of meeting the performance requirements in standalone mode. One very appealing technology for grid connected operation is tieline controls, which will regulate the active and reactive power flow between the Microgrid and the bulk grid at the point of interconnection. These controls essentially allow the Microgrid to behave as an aggregated power entity that can be made dispatchable by the utility. Particularly beneficial to the utility is the fact that this feature can be designed to compensate for intermittency associated with renewable energy resources such as wind energy and solar energy, essentially pushing the management burden inside the Microgrid. This paper reviews the overall architecture of the Microgrid concept, and presents details associated with the tieline control features.

\section{Microgrid Concept and Architecture}

A report by Navigant Consulting [1] prepared for DOE's Office of Electricity Delivery and Energy Reliability identifies four classes of Microgrids:

\section{Single Facility Microgrids}

These Microgrids include installations such as industrial and commercial buildings, residential buildings, and hospitals, with loads typically under $2 \mathrm{MW}$. These systems typically have low inertia and require backup generation for off-grid operation. Microgrids for these applications will be designed to have improved power availability and quality, and a subset of them, such as hospitals, will require a seamless transition between gridconnected and island operation.

\section{Multiple Facility Microgrids}

This category includes Microgrids spanning multiple buildings or structures, with loads typically ranging between 2 and $5 \mathrm{MW}$. Examples include campuses (medical, academic, municipal, etc), military bases, industrial and commercial complexes, and building residential developments. As with single facility Microgrids, the design of multiple facility Microgrids will be driven by the need for high availability as well as improved power quality. 


\section{Feeder Microgrids}

The feeder Microgrid will manage the generation and/or load of all entities within a distribution feeder - which can encompass 5-10MW. These Microgrids may incorporate smaller Microgrids - single or multiple facility - within them. The appeal of these Microgrids is the potential to realize regional improvements in availability, offered by the ability of the Microgrid to separate from the bulk grid during grid disturbances and service it's internal loads. Utilities, municipal utilities and coops are seen as future owners/operators of these Microgrids.

\section{Substation Microgrids}

The substation Microgrid will manage the generation and/or load of all entities connected to a distribution substation - which can encompass 5-10+MW. It will likely include some generation directly at the substation, as well as distributed generation and Microgrids included at the feeder and facility level. The appeal is again the potential to realize improvements in availability, offered by the ability of the Microgrid to separate from the bulk grid during disturbances and service its internal loads.

All of these Microgrid categories will benefit from the ability to control the dynamic exchange of power between the Microgrid and the bulk grid over the interconnecting tieline(s).

\section{Tieline Control Design}

A "tieline" refers to the feeder connection between the Microgrid and bulk grid. Tieline controls can be designed to manage the feeder power flow and voltage at the point of interconnection (POI) to meet the needs of the system operator. Control is implemented by coordinating the assets of the Microgrid, allowing the collection of these assets to appear as one aggregated dispatchable producing or consuming entity connected to the bulk grid. This section outlines the reactive and active power controls required for this capability.

\section{Microgrid Reactive Power Control (M-VAR)}

The primary functions of M-VAR are voltage regulation and power factor control at the tieline. Capabilities include voltage setpoint, steady state voltage response, and transient VAR response.

The M-VAR controller can receive either an external remote reactive power command or a voltage command from the system operator. The closed loop control issues reference VAR commands over the communication channel to each Microgrid controllable asset controller. The local controls [2] ultimately are responsible for regulating the VARs locally in each component. The controller compares the VAR output at the tieline or point of interconnection (POI) and adjusts the M-VAR command to obtain the desired system voltage. M-VAR control has two modes of operation: voltage regulated and VAR regulated (Figure 2). The voltage Vpoi refers to the measured line-to-line RMS value. Qpoi is to the total reactive power measured at the POI.
In the voltage regulation mode, the voltage error is compensated by a proportional-integral (PI) controller to produce a total reactive power demand. After subtracting the shunt reactive power, provided by the shunt capacitors (if any), the total reactive power command, Qttl,net, for the controllable asset in the Microgrid is obtained.

In the VAR regulation mode, the error between the $\mathrm{Q}$ reference and the $\mathrm{Q}$ measurement at the $\mathrm{POI}$ is regulated by a PI regulator. By adding the desired voltage feed forward, it provides a voltage reference to the voltage regulation loop. The total reactive power command is applied to the dispatch reference selection function to generate a reactive power command for each individual available controllable asset.
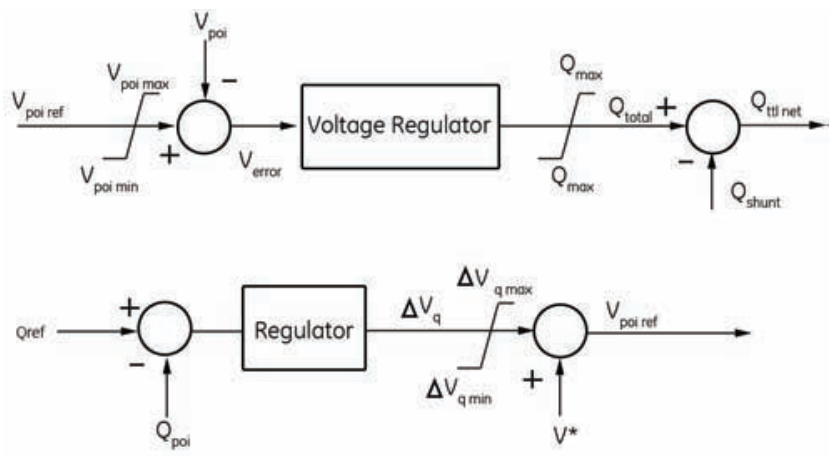

Figure 2.

M-VAR Block Diagram

\section{Microgrid Active Power Control (M-APC)}

The primary function of M-APC is to control steady-state and transient active power flow at the tieline. The objectives of the $M-A P C$ include:

- Enforcing power limits at the point of interconnection (POI)

- Enforcing ramp-rate limits at the POI

- $\quad$ Responding to system frequency excursions

These three functions are represented graphically in the block diagram in Figure 3. The parallel control loops for power limit, ramp rate limit and frequency limit will not be activated if all the operating conditions are within allowable limits. However, if any one of the controls is triggered, an adjustment command $* * * \mathrm{P}$ is generated with the intent to bring the system back to the normal operating condition. A priority is given to each parallel control loop with power limit control having highest priority and ramp rate limit control having the lowest. The total adjustment command $\star \star \star P$ is passed to the dispatch reference selection function, which allocates the ${ }^{* * *} \mathrm{P}$ among the available controllable assets based on their participation factor assigned by the optimal dispatch control. The individual adjustment is added to the P set point from the optimal dispatch control to provide the final command to the controlled assets. 


\section{Power Limit Control.}

Power limit control permits the system operator to assign a limit on the amount of active power that can be exported or imported from the grid. Power import and export are represented as negative and positive power, respectively, at the POI in the control.

\section{Power Frequency Control.}

Power frequency control is designed to support the grid frequency at the POI by adjusting active power. The inputs to the controller are the frequency and active power measured at the POI. The control law determines the power order in response to frequency excursions as specified by the system operator. A typical control law will require increased power output when frequency dips below nominal and decreased power output for increased frequency. The final output DPpr is fed to the prioritization function.

\section{Ramp Rate Limit Control.}

It is anticipated that system operators will require ramp rate control of tieline power. This control will operate by adjusting the power output of Microgrid assets to compensate for the variable nature of Microgrid loads and generation. Two rate limits are specified for both increasing and decreasing power flow. The first applies to the maximum ramp rate averaged over one minute, and the second applies to the maximum ramp rate averaged over ten minutes. The ramp rate limit calculation is designed to meet these ramp rate limits, without unnecessarily penalizing.

\section{Microgrid Energy Production.}

Power is measured at the $\mathrm{POI}$ and passed through washout filters to determine the average ramp rates. The measured ramp rates are then compared with the ramp rate limits. The resulting error signals are compared and the most limiting is selected.

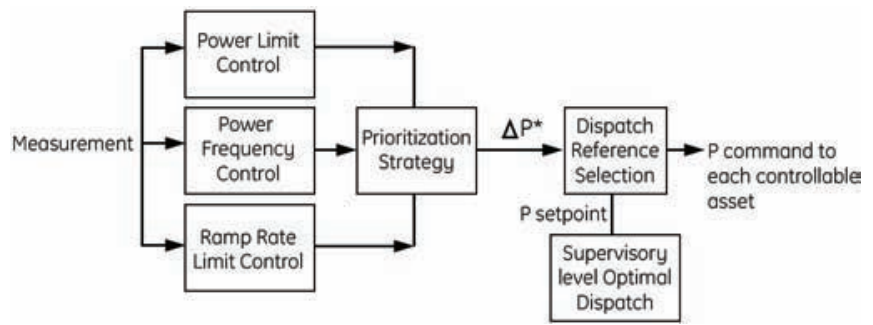

Figure 3.

M-APC Block Diagram

\section{M-APC Regulator.}

The error signals generated by power limit, ramp rate limit, and power frequency feed a prioritization block that selects a single error signal for control. This error signal is the input to a common M-APC regulator. The output of this regulator is a Microgrid power adjustment signal that is distributed to the controllable assets of the Microgrid.

\section{Case Study Results on Tieline Controls}

\section{Case Study 1: Municipal Campus Microgrid}

This case study examines a comprehensive and integrated solution to the challenge of providing reliable energy for a multifacility Microgrid.

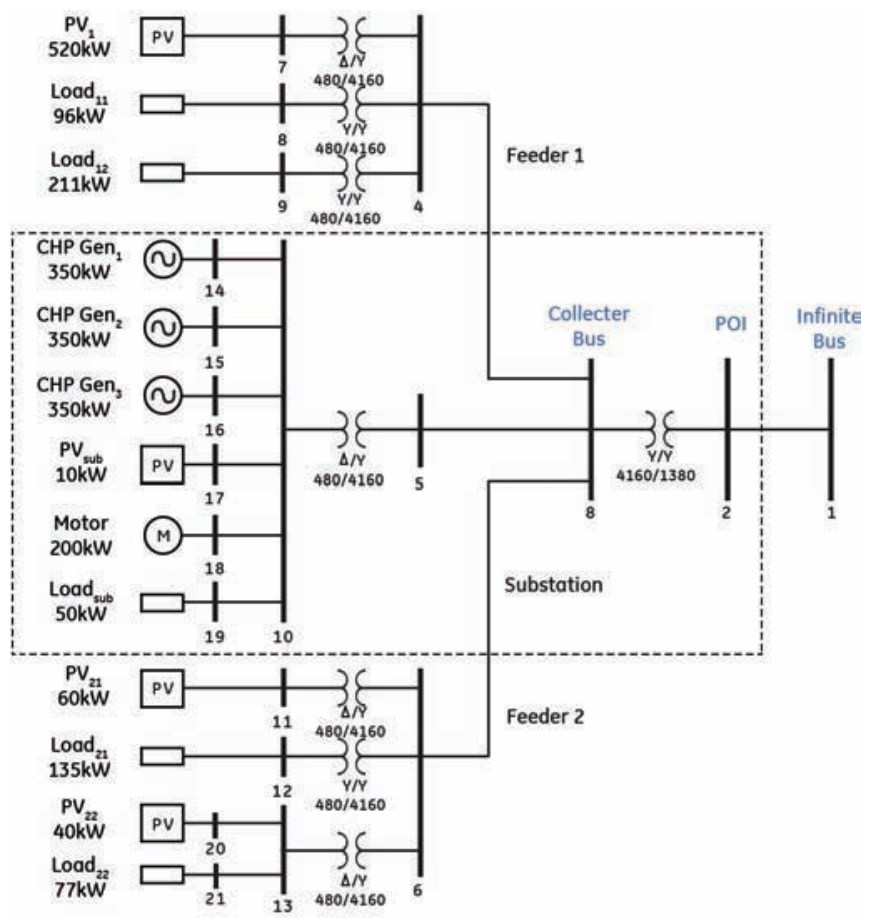

Figure 4.

Municipal Campus Microgrid

Figure 4 shows the municipal campus network considered in this example. Feeder one includes $100 \mathrm{~kW}$ of critical loads and $200 \mathrm{~kW}$ of noncritical loads and an aggregated solar PV system of $500 \mathrm{~kW}$. Feeder two includes two PV systems rated at $60 \mathrm{~kW}$ and $40 \mathrm{~kW}$ respectively, and two loads at $135 \mathrm{~kW}$ and $80 \mathrm{~kW}$. The substation houses three $350 \mathrm{~kW}$ engine gensets, a $10 \mathrm{~kW}$ solar PV system, a $250 \mathrm{KW}$ operating load, and a $250 \mathrm{~kW}$ motor load representing a chiller for CHP. The standard loads are modeled as P and Q controlled impedance loads, while the motor load is modeled as an induction motor. The solar PV system is modeled as a PV module with a DC/AC converter in $d-q$ form. The PV array in the substation is modeled with VAR control capability. The power flow in the network is solved using a traditional load flow solution, which assumes balanced (positive sequence only) conditions. Since gensets 2 and 3 are a peaker unit and a backup unit respectively. in the test cases they are both offline. Only genset 1 and the small PV at the substation are considered controllable assets. The supervisory control includes the dispatch control as well as the tieline control (M-VAR and M-APC). The goal for this case study is to analyze the control performance for tieline controls. 


\section{Impact of Voltage Disturbance}

The utility grid voltage is subject to variations that are usually within $+/-5 \%$ depending mainly on the voltage level, utility system operation and design practices. The simulation shown in Figure 5 illustrates some of the performance characteristics of the M-VAR control. M-VAR has two modes of operation: voltage regulated and VAR regulated. In this case, the system was operating under voltage control. That is, the MVAR modifies reactive power of controllable sources in order to maintain the POI voltage at its setpoint. The test consists of a $1 \%$ voltage step change at the "Infinite Bus" in Figure 4. Results are presented in Figure 5.

Shown in Figure 5 are the disturbance and variations at the POI. The transient voltage variation at the $\mathrm{POI}$ is relatively small. The reactive power variation at the $\mathrm{POI}$ is a result of the operation of the M-VAR. The active power at the POI varies due to the voltage variations inside the Microgrid. The reactive power commands to genset 1 and the PV at the substation are modified. The response time of the system is on the order of 15 seconds. This response is relatively slow compared to typical response times of excitation controls, avoiding undesirable interactions with other controls.
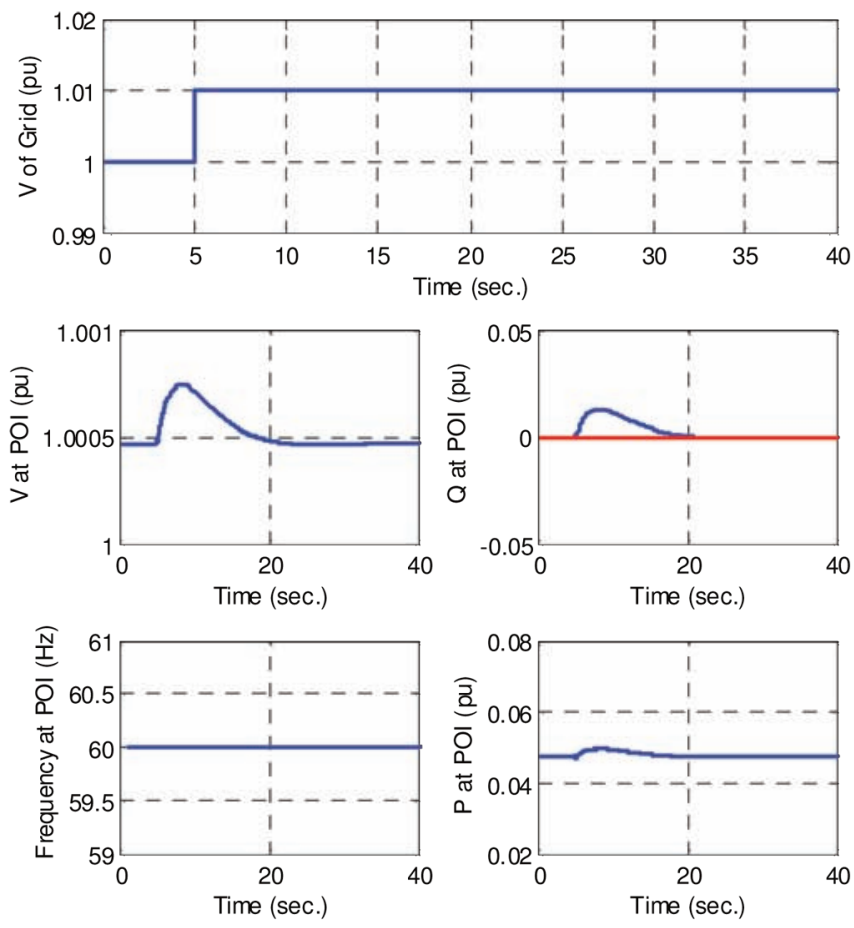

Command

\section{Response}

Figure 5.

Response at $\mathrm{POI}$ to $+1 \%$ grid voltage change

\section{Impact of Reactive Power Command Change}

To maintain voltages throughout a distribution system, a utility may send reactive power commands from the control center. A Microgrid that can meet such commands supports the system operation and provides a potential market service opportunity. The simulation shown in Figure 6 illustrates the response of the M-VAR under reactive power control to an increase in reactive power command of 0.01pu (10MW base). Figure 6 presents the simulation results.
Shown in Figure 6 are magnitudes at the POI. The reactive power at the POI follows the command with a 15 second response time. The reactive power change causes an increase in the voltage at the $\mathrm{POI}$ and inside the Microgrid, while the impact on frequency is negligible. The test results show that the controls are able to respond to this command and supply the requested reactive power at the POI by allocating it amongst the controllable generation sources. In this case, the VAR dispatchable assets are engine genset 1 and the PV at the substation.
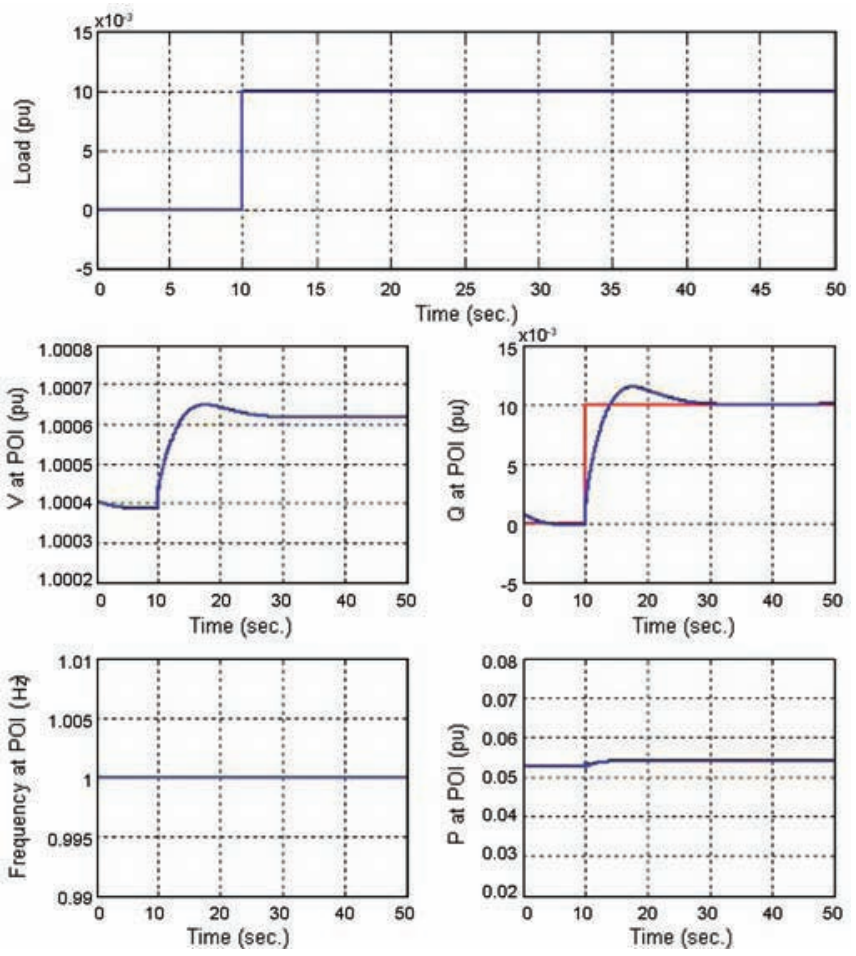

Figure 6.

Response at $\mathrm{POI}$ to $+0.01 \mathrm{pu} \mathrm{Q}$ command change

\section{Impact of Load Changes}

The total load in the Microgrid is subject to demand changes. The system should adapt to load changes to not exceed operational limits at the POI, such as power export/import limits or power ramp rate limits. The examples in this section show M-APC control under 2 scenarios:

1. A load change that exceeds the power import limit, triggering the power limit control

2. A load change causing power at the POI to ramp at a rate that exceeds the ramp rate limit, triggering the power ramp rate limit control

\section{Power Import/Export Limit.}

In this example $750 \mathrm{~kW}$ of load is ramped up in 4 seconds. The results are presented in Figure 7 . This load change causes the power import to violate the import limit at the POI. The M-APC operates to increase the power from the controllable generators to bring the active power at the POI back within limits. Genset 1 is the controllable active power source in service. The governor response of Genset 1 is significantly faster than the M-APC and 
the power output and the command almost coincide. The M-APC control was not set to operate on active power rate limitation at the POI in this example. M-VAR is in reactive power control mode and operates to compensate for the reactive power changes at the POI. Due to the load change, the system moved to a new steady state with lower voltage at the POI while maintaining the commanded Q (0 pu).
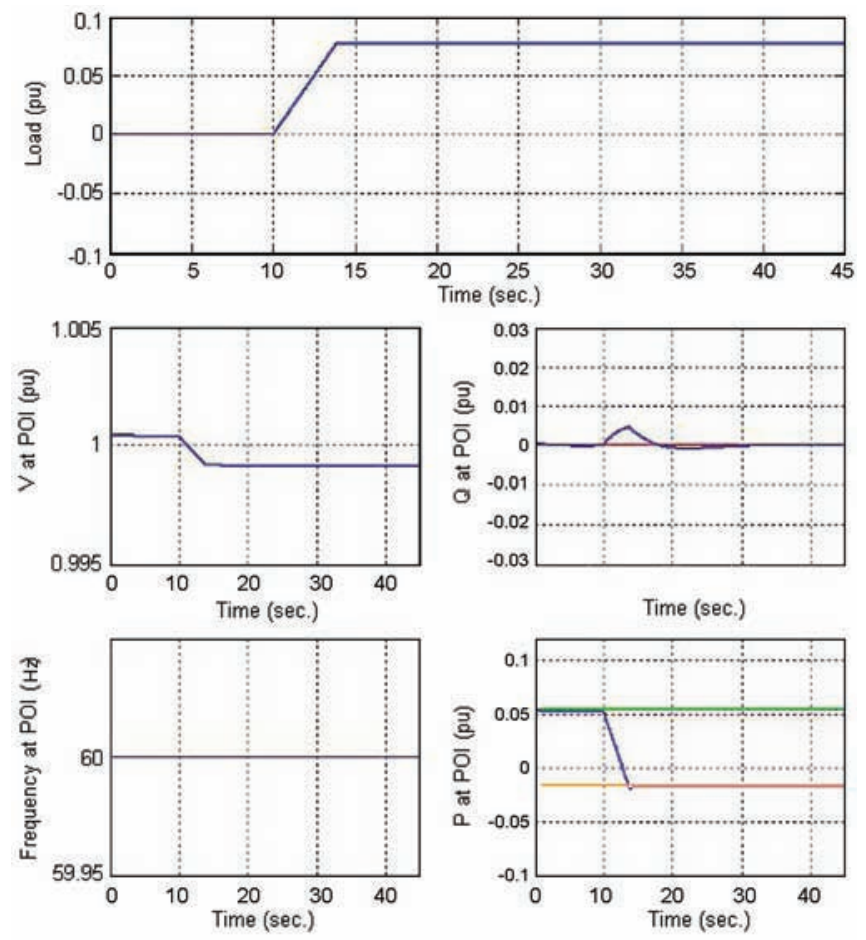

Figure 7.

Response at POI to load change (750kW) - Power Limit Control
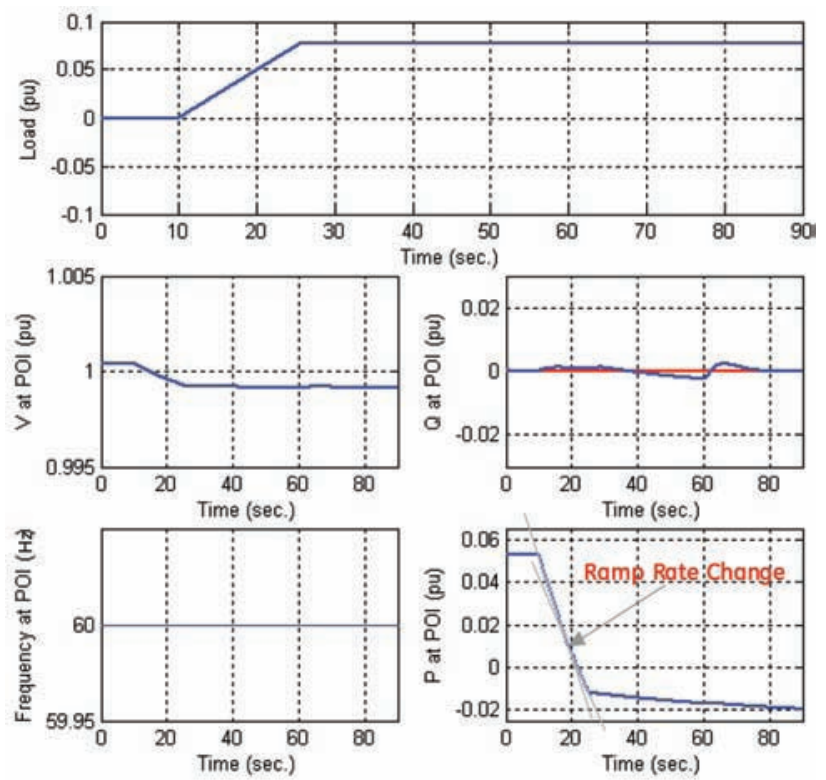

Figure 8.

Response at POI to Load change 50kW) - Power Ramp Rate Control

\section{Ramp Rate Limit.}

In this example (Figure 8), the M-APC is set to limit the ramp rate of active power at the POI. A $750 \mathrm{~kW}$ load is picked up in 15 seconds at the rate of $3 \mathrm{MW} / \mathrm{min}$, exceeding the $600 \mathrm{~kW} / \mathrm{min}$ limit at the POI. The M-APC control increases the active power output of the controllable generator to reduce the Microgrid active power rate of change. With this method of control, the Microgrid requires less active power support compared to a Microgrid without MAPC. Due to the load change, the system moved to a new steady state with lower voltage at the POI while maintaining the commanded Q 10 pu). The delta $\mathrm{P}$ command is dispatched to the only active power controllable generation, Genset 1.

\section{Case Study 2: Island Microgrid}

This second case study evaluates a potential Microgrid on an island (in the geographical sense). The model includes a $34.5 \mathrm{KV}$ line from a switching station to the Microgrid location. The network configuration is shown in Figure 9 as a single-line diagram. The model is tested with the tieline control concepts discussed in the previous section. The model includes:

- A model of a conventional run-of-river hydro generator of $500 \mathrm{~kW}$.

- A $15 \mathrm{MW}$ wind farm model represented by aggregating $10 \times$ 1.5 MW wind turbines.

- A conventional load modeled as a P \& Q controlled impedance load.

- The tieline controls, which include M-VAR and M-APC controls.

To test extreme cases, load variation sizes and power import/ export limits and ramp rate limits are assumed.

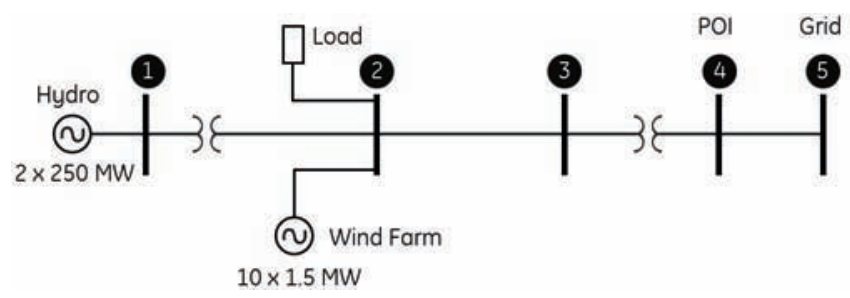

Figure 9.

Network Diagram for Case Study 2

\section{Impact of Voltage Disturbance}

A $1 \%$ voltage disturbance/step at the grid is applied at time $t=30$ seconds. This case is used to test the voltage regulation capability of the tieline control. Without a tieline controller, the voltage at the $\mathrm{POI}$ follows the disturbances and the effect of this disturbance is seen in the reactive and active powers at the POI as well as in all the wind and hydro assets (Figure 10).

M-VAR control will compensate for the voltage change at the grid side by dispatching VARs inside the Microgrid, so that the voltage at the POI will return to the setpoint after a short transient. The M-VAR control adjusts system reactive power to regulate voltage 
by commanding more VARs, allocating them among the wind and hydro. Figure 11 shows the results of a voltage disturbance at the grid, with M-VAR control and including wind variability. To show a clear response, a $2 \%$ grid voltage disturbance/step is applied in the variable wind case. Due to the variability of the wind, the control reaction in the test result is more difficult to discern, but it is clear that $\mathrm{Q}$ at the $\mathrm{POI}$ returns to its original average value. The test result shows that in the test time window, the $\sim 30 \%$ power variation from wind causes about $0.5 \%$ of voltage fluctuation at the POI with the control.
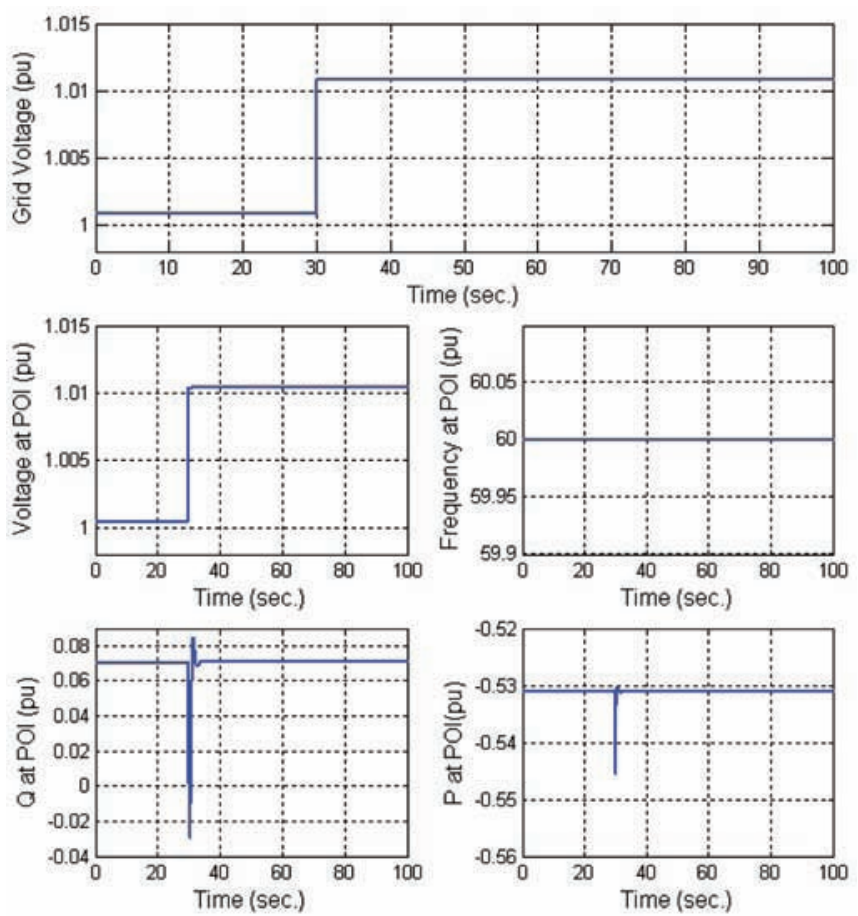

Figure 10.

Response to $1 \%$ grid voltage change - no Tieline Control, no wind variation
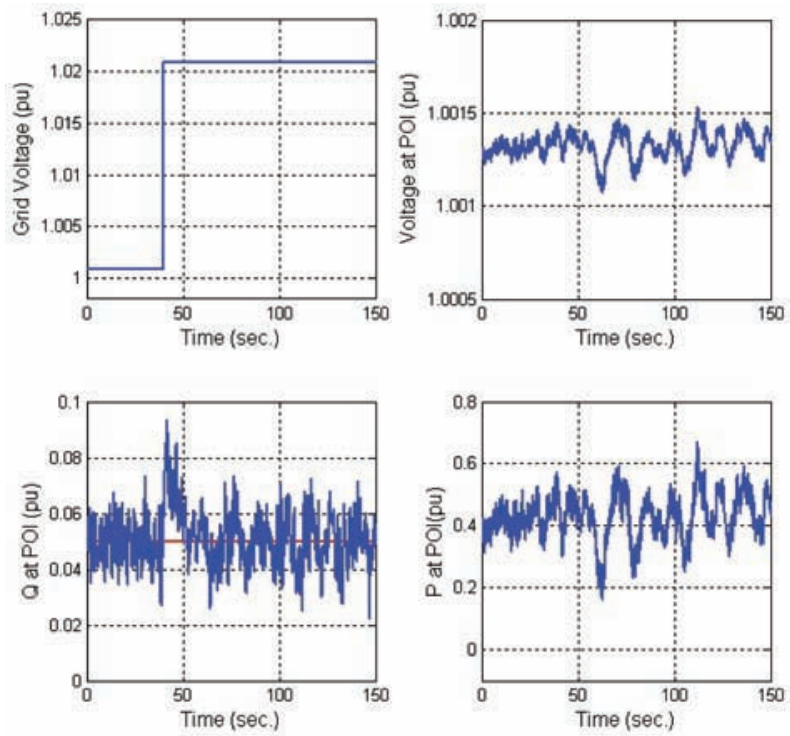

Figure 11.

Response to $2 \%$ grid voltage change with Tieline Control and wind variation

\section{Impact of Reactive Power Command Change}

Response to a reactive power commands would enable the Microgrid to provide VAR/Voltage regulation services. This test case is triggered by a $\mathrm{Q}$ command step change from an initial 500kVAR export to OkVAR export at the POI. The test was performed under constant as well as time variable wind speed conditions. The results (Figure 12) show the system responds promptly and maintains voltage stability at the POI. The simulation results with wind variability (Figure 13) show clear reactive power response but no apparent voltage or active power changes.

\section{Impact of Load Changes}

Normally, the grid would cast a limit on how much power the Microgrid can import or export, as well as how fast the change can be. This capability ensures good grid citizenship. In this example, two scenarios are tested by a load change: active power import/ export limit control and active power ramp rate control. In the first load change case (Figure 14), the load is ramped down from 10MW (1.0 pu) to 3.5MW (0.35 pu).
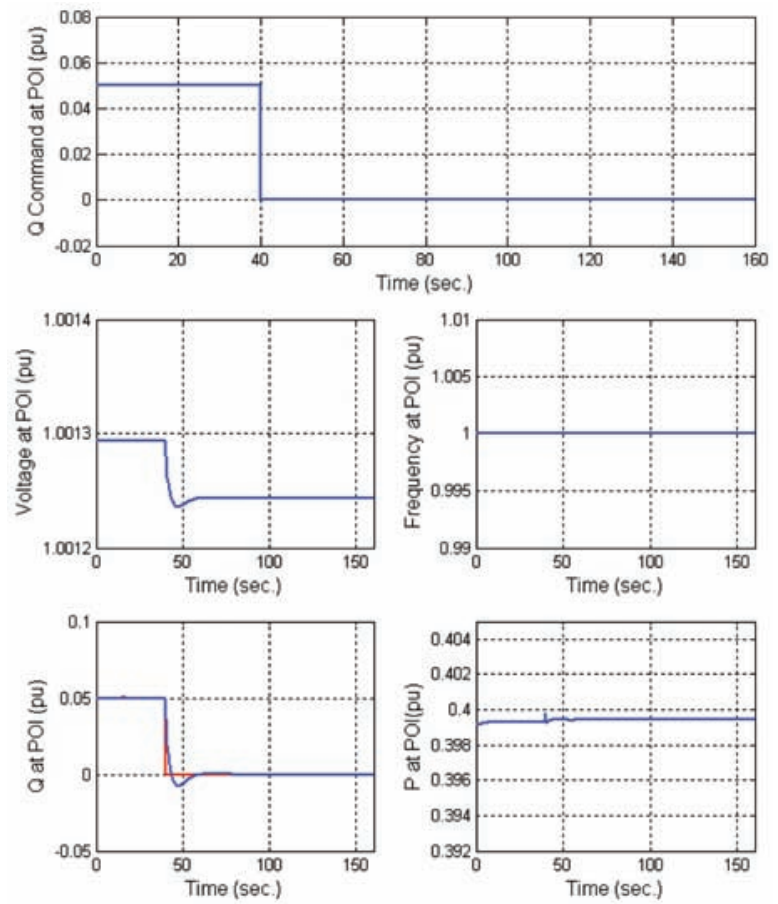

Figure 12.

Response to $Q$ command change - no wind variability
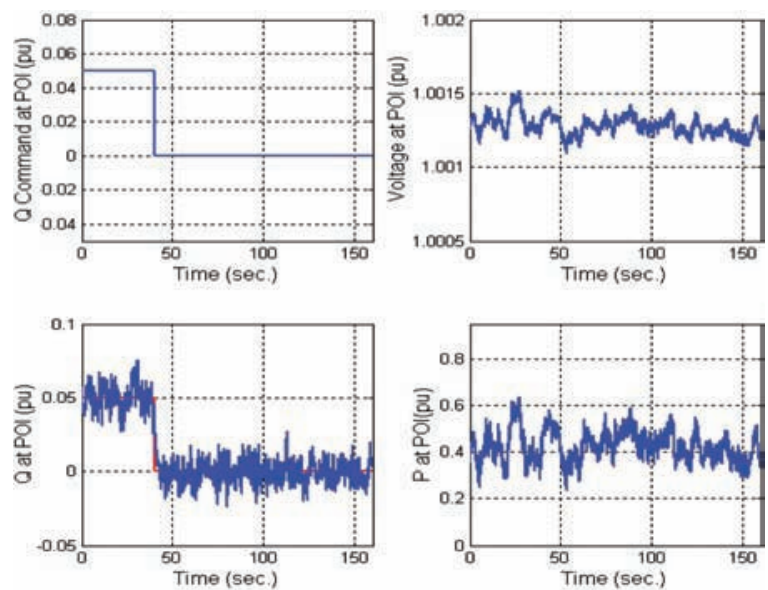

Figure 13.

Response to Q command change -wind variability 

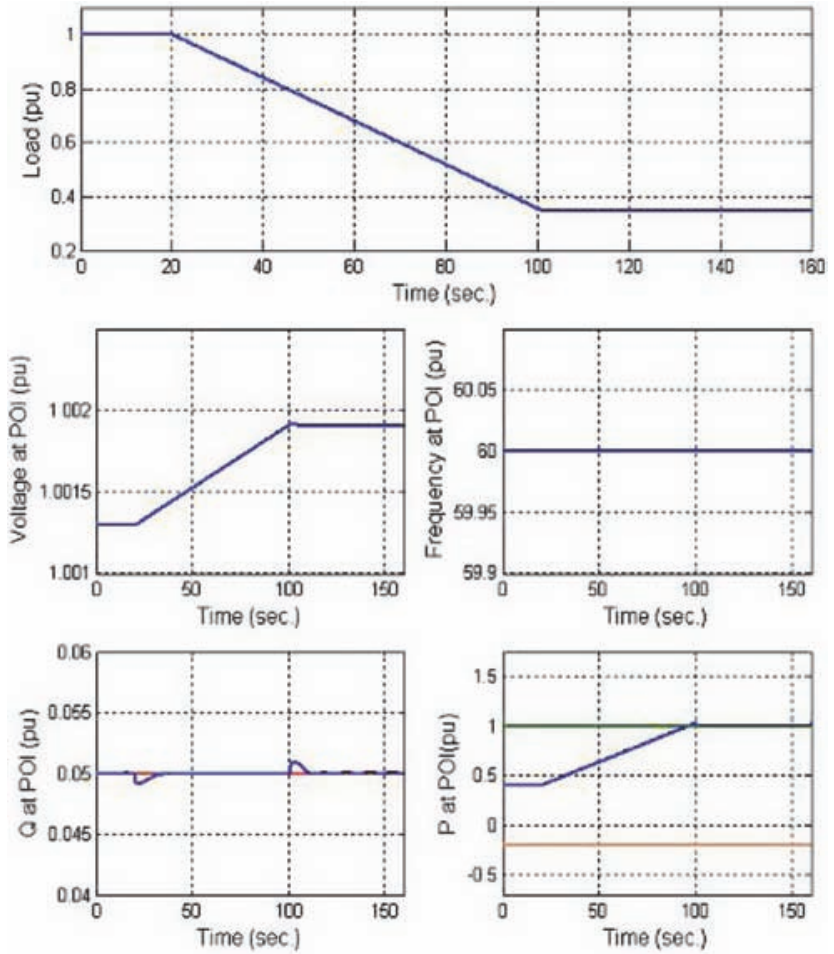

Figure 14.

Response to load change - Power Limit Control, no wind variation

The low load condition causes the power export to exceed a preset limit. As shown in the Figure, the violation triggers the M-APC, which controls the active power export so as not to exceed the limit by reducing the wind and hydro production.

Figure 15 shows the same test scenario with wind variation. The results show that meeting the power limit requirement with the fluctuation of the wind forces the hydro to be cycled more than $20 \%-30 \%$ of its capacity in a short time. This is not a desirable feature. An energy storage device may be able to take over some of the fluctuation and reduce the cycling of the hydro.
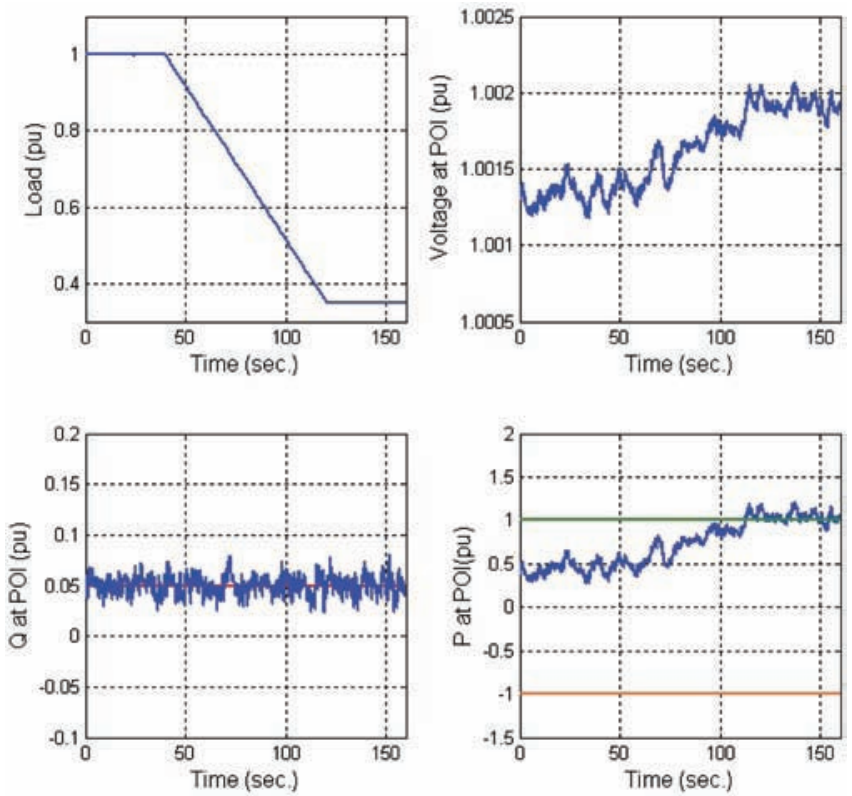

Figure 15

Response to load change - Power Limit Control, wind variation
Another load change example has the load ramped down from an initial $19 \mathrm{MW}$ to $6 \mathrm{MW}$ at a rate of $0.2 \mathrm{MW} / \mathrm{sec}$ (Figure 16). A steady wind example is shown. This causes a violation of the active power 1-minute ramp rate limit $(0.1 \mathrm{MW} / \mathrm{sec})$.

As shown in Figure 16, the ramp rate of the active power at the POI is controlled to a slower rate of change when the ramp rate limit is exceeded. The ramp rate control of M-APC limits the ramp rate by adjusting the power from each generation source. The power reduction is dispatched among the wind and hydro assets.
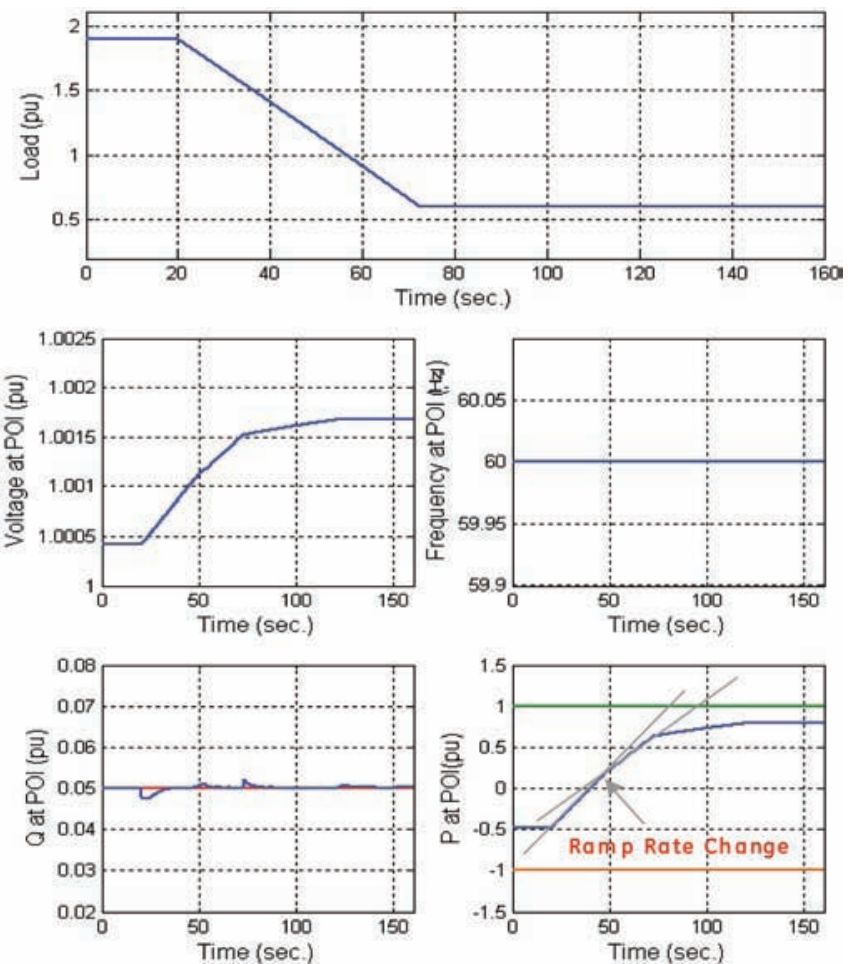

Figure 16.

Response to load change - Power Ramp Rate Control, no wind variation

\section{Lab Demonstration}

GE is working to identify a suitable centralized control hardware platform for Microgrid applications. Current lab testing employs a hardware-in-the-loop simulation of supervisory and tieline controls to validate their functionality. Figure 17 shows the layout for the laboratory setup. The setup includes four components:

- Single board computer (SBC) rack with QNX real-time operating system (RTOS), +/- $10 \mathrm{~V}$ analog input and output cards.

- $\quad$ RT-LAB software coupled with generation and load asset models for the Microgrid.

- Supervisory controls developed using Simulink and linked with an OLE for Process Control (OPC) interface.

- GE Universal Relay (UR). The GE UR family [3] is a new generation of modular relays built on a common platform. The UR features high performance protection and communications. 
RT-LAB software is used in conjunction with Simulink's Real-Time Workshop to compile the generation and load asset models into $C$ code that can then be downloaded to the single board computer rack. These models consists of two generators and five loads that can be connected or disconnected to the system and a bus network that is connected to the grid. This model also includes drivers to interface with the digital-to-analog (D/A) and analogto-digital (A/D) cards connected to the rack. The $A / D$ card receives the active and reactive power commands for each generator from the UR as a voltage that is then scaled to the appropriate per unit value via a gain multiplier. The D/A card sends the scaled power and reactive power output of each generator, the reactive and active power at POI and the POI voltage and frequency to the UR.

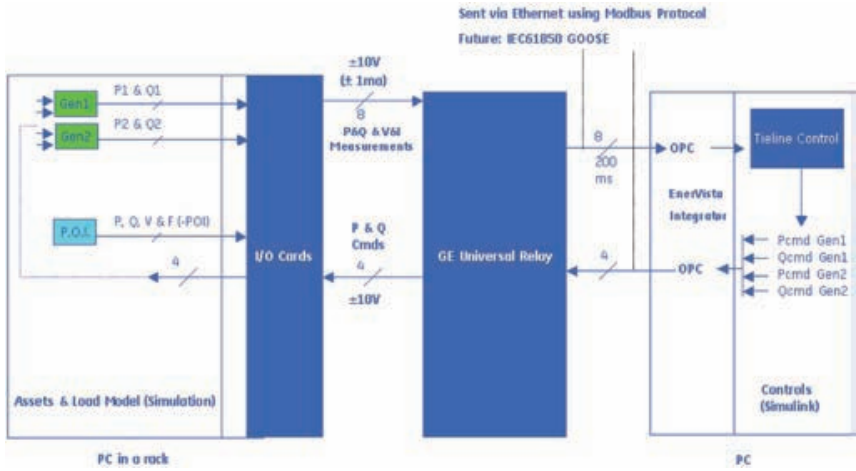

Figure 17.

Lab Setup Block Diagram

The UR is the hardware interface between the supervisory controls and generators. The analog measurements from the generators and POI are sent via Ethernet and IEC 61850 GOOSE/ OPC to the supervisory controls in Simulink. The supervisory controls recalculate the active and reactive power commands for each generator based on the current state of the generators and the POI. The new commands are then sent to the UR where they are scaled to a voltage that represents the analog value of the commands. The voltage is sensed by the SBC rack's analog input card and the generators adjust their output accordingly.

\section{Lab Testing Results}

The lab test shown in this section utilizes the electrical model developed for the Municipal Campus Microgrid described earlier and represented by Figure 4 . This test case examines the system response after disconnecting a load from the Microgrid. The maximum connected Microgrid load is equal to $770 \mathrm{~kW}$. The goal of the test is to confirm the functionality of the supervisory controls after the controls and generators have been separated into separate subsystems that interface with analog and OPC signals. In the test, a 0.03 pu load is disconnected from the Microgrid. The results of the test are shown in Figure 18.

The generator responds to the active power command and behaves as expected. The M-APC increases the power from the controllable generator (Gen1) to keep the active power at the POI within the defined limit. The power flow at the POI indicates that power is now exported to the grid after the load is disconnected.
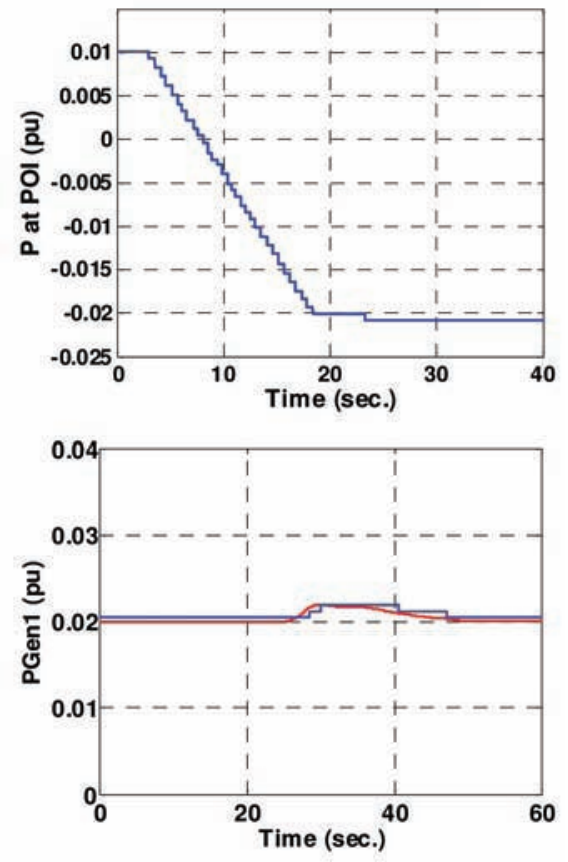

Figure 18.

Response to $30 \mathrm{~kW}$ load step change

\section{Conclusions}

This paper presents details on an important Microgrid control feature: tieline controls. These controls essentially coordinate the response of the several distributed energy resources within the Microgrid, such as generation, energy storage, or controllable loads, to make the response of the aggregate system at the point of interconnection to the grid resemble one single dispatchable entity. As such, the Microgrid can become a better citizen to the grid, managing its power exchange with the grid, and supporting voltage at the point of interconnection. One interesting benefit of this technology is that it can be designed to compensate for intermittency associated with renewable energy resources such as wind energy and solar energy, pushing the intermittency management burden inside the Microgrid, thereby potentially allowing for an increased penetration of these renewable energy resources. The dynamic response of the tieline control active and reactive power compensation elements were illustrated in several simulations, as well as in a hardware-in-the-loop simulation environment.

\section{References}

[1] Navigant Consulting Inc., "Final Report Microgrids Research Assessment for the US Department of Energy's Office of Electricity Delivery and Energy Reliability and the California Energy Commission's Public Interest Energy Research Program", May 2006.

[2] Nicholas W. Miller, Einar V. Larsen, and Jason M. MacDowell, "Advanced Control of Wind Turbine-Generators to Improve Power System Dynamic Performance", 11th International Conference on Harmonics and Quality of Power, 2004.

[3] Brochure for N60 Network Stability and Synchrophasor Measurement System: http://www.geindustrial.com/cwc 